Research article

Open Access

\title{
Involvement of a specificity proteins-binding element in regulation of basal and estrogen-induced transcription activity of the BRCA1 gene
}

\author{
Jennifer K Hockings ${ }^{1,2}$, Stephanie C Degner ${ }^{2}$, Sherif S Morgan², Michael Q Kemp² and \\ Donato F Romagnolo1,2
}

\begin{abstract}
${ }^{1}$ Cancer Biology Interdisciplinary Graduate Program, Department of Nutritional Sciences, The University of Arizona, E 4th Street, Tucson, Arizona 85721-0038, USA

2Laboratory of Mammary Gland Biology, Department of Nutritional Sciences, The University of Arizona, E 4th Street, Tucson, Arizona 85721-0038, USA
\end{abstract}

Corresponding author: Donato F Romagnolo, donato@u.arizona.edu

Received: 11 Sep 2007 Revisions requested: 22 Oct 2007 Revisions received: 28 Feb 2008 Accepted: 31 Mar 2008 Published: 31 Mar 2008

Breast Cancer Research 2008, 10:R29 (doi:10.1186/bcr1987)

This article is online at: http://breast-cancer-research.com/content/10/2/R29

(c) 2008 Hockings et al.; licensee BioMed Central Ltd.

This is an open access article distributed under the terms of the Creative Commons Attribution License (http://creativecommons.org/licenses/by/2.0), which permits unrestricted use, distribution, and reproduction in any medium, provided the original work is properly cited.

\begin{abstract}
Introduction Increased estrogen level has been regarded to be a risk factor for breast cancer. However, estrogen has also been shown to induce the expression of the tumor suppressor gene, $B R C A 1$. Upregulation of BRCA1 is thought to be a feedback mechanism for controlling DNA repair in proliferating cells. Estrogens enhance transcription of target genes by stimulating the association of the estrogen receptor (ER) and related coactivators to estrogen response elements or to transcription complexes formed at activator protein (AP)-1 or specificity protein (Sp)-binding sites. Interestingly, the BRCA1 gene lacks a consensus estrogen response element. We previously reported that estrogen stimulated BRCA1 transcription through the recruitment of a p300/ER- $\alpha$ complex to an AP-1 site harbored in the proximal BRCA1 promoter. The purpose of the study was to analyze the contribution of cis-acting sites flanking the AP-1 element to basal and estrogen-dependent regulation of BRCA1 transcription.
\end{abstract}

Methods Using transfection studies with wild-type and mutated BRCA1 promoter constructs, electromobility binding and shift assays, and DNA-protein interaction and chromatin immunoprecipitation assays, we investigated the role of Spbinding sites and cAMP response element (CRE)-binding sites harbored in the proximal BRCA1 promoter.
Results We report that in the BRCA1 promoter the AP- 1 site is flanked upstream by an element (5'-GGGGCGGAA-3') that recruits Sp1, Sp3, and Sp4 factors, and downstream by a half CRE-binding motif (5'-CGTAA-3') that binds CRE-binding protein. In ER- $\alpha$-positive MCF-7 cells and ER- $\alpha$-negative Hela cells expressing exogenous ER- $\alpha$, mutation of the Sp-binding site interfered with basal and estrogen-induced BRCA1 transcription. Conversely, mutation of the CRE-binding element reduced basal BRCA1 promoter activity but did not prevent estrogen activation. In combination with the AP-1/CRE sites, the Sp-binding domain enhanced the recruitment of nuclear proteins to the BRCA1 promoter. Finally, we report that the MEK1 (mitogen-activated protein kinase kinase-1) inhibitor PD98059 attenuated the recruitment of Sp1 and phosphorylated ER- $\alpha$, respectively, to the Sp and AP-1 binding element.

Conclusion These cumulative findings suggest that the proximal BRCA1 promoter segment comprises cis-acting elements that are targeted by Sp-binding and CRE-binding proteins that contribute to regulation of BRCA1 transcription.

\section{Introduction}

Gene expression in mammary tissue is under the control of ovarian steroids, including estrogen, which regulates tran- scription activity of target promoters by stimulating the recruitment of either the estrogen receptor (ER)- $\alpha$ or ER- $\beta$ [1]. In the classical pathway, the liganded ER- $\alpha$ homodimerizes and

$\overline{\mathrm{AP}}=$ activator protein; ChIP = chromatin immunoprecipitation; CRE = cAMP response element; CREB = CRE-binding protein; DMEM = Dulbecco's modified Eagle's medium; ER = estrogen receptor; ERE = estrogen response element; FBS = fetal bovine serum; MAPK = mitogen-activated protein kinase; MEK = MAPK kinase; PCR = poolymerase chain reaction; Sp = specificity protein. 
binds directly to DNA at estrogen response elements (EREs) [2]. The physical contact of the ER- $\alpha$ homodimer with DNA facilitates the subsequent recruitment of coregulators [3]. The latter factors contribute to chromatin reorganization and transcriptional coactivation [4]. Alternatively, the ER- $\alpha$ can physically interact with DNA-bound protein complexes containing, among other factors, the activator protein (AP)-1 [5], cAMP response element (CRE [TGACGTCA])-binding protein (CREB) [1], or specificity protein (Sp) family members [6,7]. The $\mathrm{Sp}$ factors have been shown to bind directly to the consensus GC-rich region 5'-(G/T)GGGCGG(G/A)(G/A)-3' or DNA-bound AP-1 transcription complexes [8].

Members of the Sp family of transcription factors exert differential effects on gene transcription. For example, Sp1 and Sp4 have been shown to act as transcriptional activators, whereas Sp3 was reported to antagonize Sp1 activation functions by competing for promoter occupancy [9-11]. The Sp family members play a significant role in tissue and organ development, because mice lacking at least one of the Sp proteins exhibit various physiological abnormalities, including embryonic lethality [12], growth retardation [13,14], and sterility [15]. Increased expression of Sp1 has been detected in gastric $[16,17]$ pancreatic [18], and breast [19] tumors. In addition to inducing the recruitment of ER to target promoters, estrogen influences gene expression through the activation of mitogen-activated protein kinases (MAPKs), which phosphorylate the ER- $\alpha$ and other transcription factors including Sp1 [20] and AP-1 [2]. Phosphorylation of these factors increases their binding affinity for cognate DNA elements or DNA-bound coregulators [21].

The breast cancer susceptibility gene BRCA1 encodes a phosphoprotein that is involved in the DNA damage response $[22,23]$ and regulation of cell cycle checkpoints [24]. About $30 \%$ to $40 \%$ of sporadic breast cancers, which represent the vast majority (90\% to $95 \%$ ) of breast cancer cases, exhibit lower or absent levels of BRCA1 in the absence of mutations in the BRCA1 gene $[25,26]$. Because altered regulation of $B R C A 1$ may increase the likelihood of developing breast cancer, it is important to examine the molecular events that regulate normal BRCA1 expression.

In proliferating breast epithelial cells, the cellular levels of BRCA1 mRNA and protein have been shown to increase in response to estrogen [27-29]. The BRCA1 promoter harbors two distinct transcriptional start sites (exon-1A and exon-1B). However, translation of BRCA1 mRNA always starts from the ATG codon located on exon-2 [30]. Upregulation of BRCA1 expression in response to estrogen may represent a feedback mechanism that represses ER-signaling during the early stages of breast tumorigenesis [31]. This interpretation is supported by evidence indicating that the BRCA1 protein represses the transcriptional activity of the liganded ER- $\alpha$ and estrogen-regulated genes [32]. However, the BRCA1 gene appears not to be regulated by estrogen through the classical pathway, because the BRCA1 promoter lacks canonical EREs. Recently, our laboratory reported that estrogen stimulated $B R C A 1$ promoter activity by inducing the recruitment of an ER- $\alpha /$ p300 transcription complex to an AP-1 element in close proximity to the transcriptional start site of exon-1B $[33,34]$. The objective of the present work was to elucidate the roles played by $\mathrm{Sp}$-binding and CRE-binding elements flanking the AP-1 site in regulation of BRCA1 transcription.

\section{Materials and methods}

The experimental research reported in the manuscript has been performed with the approval of the Institutional Biosafety Committee of the University of Arizona (Tucson, AZ, USA), on 20 December 2005.

\section{Cell culture and chemicals}

MCF-7 and HeLa cells were obtained from the American Type Culture Collection (Manassas, VA, USA) and maintained in Dulbecco's modified Eagle's medium (DMEM)/F12 (SigmaAldrich Chemical Co., St. Louis, MO, USA), supplemented with 10\% fetal bovine serum (FBS; Hyclone Laboratories, Logan, UT, USA) as described previously [29]. Estrogen, penicillin/streptomycin solution, and DMEM/F12 were purchased from Sigma-Aldrich Chemical Co. Antibodies against ER- $\alpha$, Sp1, Sp3, and Sp4 were purchased from Santa Cruz Biotechnologies, Inc. (Santa Cruz, CA, USA). The antibody against phosphorylated ER- $\alpha$ (Ser118) was obtained from Cell Signaling (Beverly, MA, USA).

\section{Site-directed mutagenesis}

Details concerning the cloning of a 1.7-kilobase BRCA1 promoter fragment into pGL3 Basic (pGL3BRCA1) are described elsewhere [35]. Mutation of the Sp (GGGGCGG to GCTAAG) and half-CRE (CGTAA to CtgcA) core sequence in the BRCA1 promoter was carried out by sitedirected mutagenesis (Stragene, La Jolla, CA, USA) using the following primers synthesized by Sigma-Genosys (The Woodlands, TX, USA): Sp-F-Mut: 5'-GGGTAGGctaaGAACCTGAGAGGCGTAAGG CG-3'; Sp-R-Mut: 5'CGCCTTACGCCTCTCA GGTTCttagCCTACCC-3'; CRE-FMut: 5'-GGAACCTGAGAGGCtgcAGGCGTTGTGAAG-3'; and CRE-R-Mut: 5'-CTTCACAACGCCTgcaGCCTCTCAGGTTCC-3'. The insertion of mutations was confirmed by direct sequencing.

\section{Transfection studies}

Experimental conditions for cell culture and transfection of reporter plasmids were those described previously [33]. Briefly, MCF-7 and HeLa cells were cultured for 3 days in phenol red-free DMEM/F12 and supplemented with 5\% charcoalstripped FBS. Then, cells were seeded in six-well plates 24 hours before transfection using the Lipofectamine-Plus (Invitrogen, Carlsbad, CA, USA). Plasmids encoding renilla were also cotransfected to account for variations in transfection 
efficiency and cell density. Luciferase reporter activity was monitored with a Luminometer 20/20 (Turner Biosystems, Sunnyvale, CA, USA) and expressed as relative luciferase units corrected for renilla.

\section{DNA-protein pull-down assay}

Eighty to ninety per cent confluent breast MCF-7 cells were cultured for 3 days in phenol red-free DMEM/F12 and supplemented with $5 \%$ charcoal-stripped FBS. Cells were then maintained in phenol red-free DMEM/F12 plus vehicle with $5 \%$ charcoal-stripped FBS with or without $10 \mathrm{nmol} / \mathrm{l}$ estrogen before nuclear extracts were obtained using the Nuclear/Cytoplasmic Extract Kit and quantitated using the BCA protein assay (Pierce Biotechnology, Rockford, IL, USA). The biotinlabeled double stranded oligonucleotides were based on the human BRCA1 promoter sequence. The DNA-protein pulldown assay was performed as described previously [36]. Briefly, MCF-7 nuclear extracts were mixed with biotin-labeled oligonucleotides and streptavidin agarose beads with $70 \%$ slurry. After 2 hours of incubation at room temperature, beads were centrifuged and washed with cold phosphate buffer saline. Bound proteins were then separated on $4 \%$ to $12 \%$ PAGE and Western blot analysis was performed with antibodies against Sp1, Sp3, total ER- $\alpha$, or phosphorylated ER- $\alpha$. Oligonucleotides used for the pull-down assay were as follows: Sp/AP-1, forward: 5'-GGGTAGGGGCGGAACCTGAGAGGCGTAA-3'; Sp/AP-1, reverse: 5'-TTACGCCTCTCAGGTTCCGCCCCTACCC-3'; Sp-Mut/AP-1, forward: 5'GGGTAGGctaaGAACCTGAGAGGCGTAA-3'; and Sp-Mut/ AP-1, reverse: 5'-TTACGCCTCTCAGGTTCttagCCTACCC3 '.

\section{Electrophoretic mobility binding and shift assays}

The procedure used for electromobility binding assay was described previously [33]. Cells were plated in 6-well Costar tissue culture plates in DMEM plus 5\% charcoal-stripped FBS. After 24 hours, cells were cultured in control DMEM plus vehicle or $10 \mathrm{nmol} / \mathrm{l}$ estrogen. Then, cells were trypsinized and washed with ice-cold phosphate buffer saline. Cells were resuspended in ice-cold $25 \mathrm{mmol} / \mathrm{l}$ Hepes buffer containing $1.5 \mathrm{mmol} / \mathrm{I}$ EDTA, $1 \mathrm{mmol} / \mathrm{I} \mathrm{DTT}, 0.5 \mathrm{mmol} / \mathrm{PMSF}$ and $5 \mu \mathrm{g} /$ $\mathrm{ml}$ aprotinin, and placed on ice for 10 minutes. Cells were pelleted and resuspended in $1 \mathrm{ml}$ ice-cold $25 \mathrm{mmol} / \mathrm{l}$ Hepes buffer containing $1.5 \mathrm{mmol} / \mathrm{l}$ EDTA, 10\% (vol/vol) glycerol, 1 $\mathrm{mmol} / \mathrm{I}$ DTT, $0.5 \mathrm{mmol} / \mathrm{l} \mathrm{PMSF}$, and $5 \mu \mathrm{g} / \mathrm{ml}$ aprotinin. The cell suspension was transferred to a mortar for drilling with a Teflon pestle until more than $90 \%$ of the cells in a $2 \mu$ aliquot were unable to exclude trypan blue. After centrifugation, cell pellets were resuspended in $150 \mu$ ice-cold $25 \mathrm{mmol} / \mathrm{l}$ Hepes buffer containing $1.5 \mathrm{mmol} / \mathrm{EDTA}, 10 \%$ (vol/vol) glycerol, 0.5 $\mathrm{mol} / \mathrm{l} \mathrm{KCl}, 1 \mathrm{mmol} / \mathrm{I} \mathrm{DTT}, 0.5 \mathrm{mmol} / \mathrm{L}$ PMSF and $5 \mu \mathrm{g} / \mathrm{ml}$ aprotinin, and placed on ice with intermittent vortexing. Cell debris was removed by centrifugation. Supernatants containing nuclear protein were stored at $-70^{\circ} \mathrm{C}$. Nuclear protein concentration was determined using the BCA protein assay (Pierce
Chemical Company). Oligonucleotides used for binding and shift assays are summarized in Table 1. The complementary oligonucleotides were annealed then phosphorylated at the 5'end with $\left[\gamma^{-32}\right.$ P]ATP and T4 polynucleotide kinase. Unincorporated nucleotides were removed using the TE-10 spin columns (Clontech Laboratories Inc., Mountain View, CA, USA). Binding assays were performed by incubating $5 \mu \mathrm{g}$ nuclear protein in the binding buffer then incubated with the labeled oligonucleotides for 20 minutes. For supershift assays, antibodies (Affinity Bioreagents, Golden, CO, USA) were incubated with $1 \mu \mathrm{g}$ nuclear extracts for 2 hours before addition of labeled oligonucleotides. For cold competition, a 100-fold excess of the respective unlabeled oligonucleotides was added to the binding reaction 10 minutes before addition of the labeled oligonucleotides. For supershift assays, antibodies (Santa Cruz Biotechnologies) were incubated with $1 \mu \mathrm{g}$ nuclear extracts for 2 hours before addition of labeled oligonucleotides. For cold competition, a 100-fold excess of the respective unlabeled oligonucleotides was added to the binding reaction 10 minutes before addition of the labeled oligonucleotides. Samples were electrophoresed through a 5\% nondenaturing polyacrylamide gel at $200 \mathrm{~V}$ for 90 minutes. Finally, the gel was dried and exposed to a phosphor screen, and digital phosphorimages were retrieved using the Storm system (Molecular Dynamics, Sunnyvale, CA, USA).

\section{Chromatin immunoprecipitation assay and real-time PCR}

Chromatin immunoprecipitation assay and real-time PCR were carried out as described previously [34,37]. Briefly, MCF-7 cells were prepared in phenol red-free DMEM/F12 supplemented with $5 \%$ charcoal-stripped FBS for 3 days. After treatment with control DMEM plus vehicle or $10 \mathrm{nmol} / \mathrm{l}$ estrogen, chromatin was crosslinked with formaldehyde (1\% final concentration) for 10 minutes at room temperature and subsequently glycine was added to quench the formaldehyde crosslinking. Cells were then harvested and resuspended in lysis buffer (1\% SDS, $10 \mathrm{mmol} / \mathrm{I}$ EDTA, $50 \mathrm{mmol} / \mathrm{l} \mathrm{Tris}-\mathrm{HCl}$, and protease inhibitor cocktail). After sonication (10 $\times 15 \mathrm{sec}-$ onds), samples were diluted in chromatin immunoprecipitation (ChIP) buffer (1\% Triton X-100, 2 mmol/I EDTA, $150 \mathrm{mmol} / \mathrm{l}$ $\mathrm{NaCl}, 20 \mathrm{mmol} / \mathrm{l}$ Tris- $\mathrm{HCl}$, and protease inhibitor cocktail). Dilutions of chromatin preparations were reserved as either input (no antibody) or used for immunoprecipitation with the desired antibody or $\lg G$ as a negative control. The chromatin immunoprecipitation was conducted utilizing the Magna ChIP kit (17-611) obtained from Millipore (Billenca, MA, USA), in accordance with the protocol provided. Briefly, the sonicated samples were immunoprecipitated overnight at $4^{\circ} \mathrm{C}$ with the antibodies and $\mathrm{G}$ magnetic beads. The magnet beads were pelleted and sequentially washed with a low salt immune complex wash buffer, high salt immune complex wash buffer, $\mathrm{LiCl}$ immune complex wash buffer, and TE buffer. The protein-DNA complexes were eluted and crosslinking was reversed by incubation with ChIP elution buffer and proteinase $\mathrm{K}$ for $2 \mathrm{~h}$ at 
Table 1

BRCA-1 Oligonucleotides used for binding and shift assays

\begin{tabular}{|c|c|}
\hline Promoter element & Oligonucleotides \\
\hline \multirow[t]{2}{*}{ AP-1/CRE } & Sense: 5'-AACCTGAGAGGCGTAAGGCGT-3', \\
\hline & Antisense: 5'-ACGCCTTACGCCTCTCAGGTT-3'; \\
\hline \multirow[t]{2}{*}{ Sp/AP-1/CRE } & Sense: 5'-GGGTAGGGGCGGAACCTGAGAGGCGTAAGGCGT-3', \\
\hline & Antisense: 5'-ACGCCTTACGCCTCTCAGGTTCCGCCCCTACCC-3'; \\
\hline \multirow[t]{2}{*}{ Sp-Mut/AP-1/CRE } & Sense: 5'-GGGTAGGctaaGAACCTGAGAGGCGTAAGGCGT-3', \\
\hline & Antisense: 5'-ACGCCTTACGCCTCTCAGGTTCttagCCTACCC-3' \\
\hline \multirow[t]{4}{*}{ Sp/AP-1-Mut/CRE } & Sense: 5'-GGGTAGGGGCGGAACtatAGAGGCGTAAGGCGT-3', \\
\hline & Antisense: 5'-ACGCCTTACGCCTCTataGTTCCGCCCCTACCC-3'; \\
\hline & Sense: 5'-GGGTAGGGGCGGAACCactaAGGCGTAAGGCGT-3', \\
\hline & Antisense: 5'-ACGCCTTACGCCTtagtGGTTCCGCCCCTACCC-3'; \\
\hline \multirow[t]{4}{*}{ Intervening AP-1/CRE sequences: } & Sense: 5'-GGGTAGGGGCGGAACCTGAtctGCGTAAGGCGT-3', \\
\hline & Antisense: 5'-ACGCCTTACGCagaTCAGGTTCCGCCCCTACCC-3'; \\
\hline & Sense: 5'-GGGTAGGGGCGGAACCTGAGAttaGTAAGGCGT-3', \\
\hline & Antisense: 5'-ACGCCTTACtaaTCTCAGGTTCCGCCCCTACCC-3'; \\
\hline \multirow[t]{4}{*}{ Sp/Ap-1/CRE-Mut } & Sense: 5'-GGGTAGGGGCGGAACCTGAGAGGCtgcAGGCGT-3', \\
\hline & Antisense: 5'-ACGCCTgcaGCCTCTCAGGTTCCGCCCCTACCC-3'; \\
\hline & Sense: 5'-GGGTAGGGGCGGAACCTGAGAGGCGTcttGCGT-3', \\
\hline & Antisense: 5'-ACGCaagACGCCTCTCAGGTTCCGCCCCTACCC-3'; \\
\hline
\end{tabular}

$A P$, activator protein; $C R E$, cAMP response element; $S p$, specificity protein.

$62^{\circ} \mathrm{C}$, followed by incubation for 10 minutes at $95^{\circ} \mathrm{C}$. The free DNA was then purified using the provided spin columns.

PCR primers used to amplify the BRCA1 promoter region flanking the Sp/AP-1/CRE binding sites were as follows: forward: 5'-CTGACAGATGGGTATTCTTTGACG-3'; and reverse: 5'-GCATATTCCAGTTCC TATCACGAG-3' (171 base pairs). The iTaq SYBR Green Supermix reagents (BioRad, Hercules, CA, USA) were used as described by the manufacturer. Reactions were run at a final volume of $25 \mu \mathrm{l}$ consisting of $12.5 \mu \mathrm{l}$ of $2 \times$ SYBR Green Supermix with ROX (0.4 $\mathrm{mmol} / \mathrm{l}$ each of dATP, dCTP, dGTP, and dTTP, $50 \mathrm{U} / \mathrm{ml}$ iTaq DNA polymerase, $6 \mathrm{mmol} / \mathrm{Mgg}^{2+}$, SYBR Green I, and ROX reference dye), $1 \mu$ leach of forward and reverse primers added at a final concentration of $200 \mathrm{nM}, 2 \mu \mathrm{LNA}$, and $8.5 \mu \mathrm{l}$ nuclease-free double-distilled water. The $A B I 5700$ sequence detection system and comparative $C_{T}$ method were used to quantify the relative differences in PCR. BRCA1 promoter amplicons were normalized to input.

\section{Statistical analysis}

Results of transfection studies and real-time PCR from ChIP assays are presented as means \pm standard error of the mean. Statview, the SAS Institute (Cary, NC, USA) statistical analysis software was used for analysis of variance. Comparison of means following a significant $(P<0.05)$ analysis of variance test was performed by Fisher's protected least significant difference test.

\section{Results}

The primary objective of this study was to examine whether cis-acting elements flanking an AP-1 site located in the proximal BRCA1 promoter played a role in the basal and estrogendependent regulation of BRCA1 transcription. We mapped immediately upstream from the AP-1 site a $\mathrm{GC}$-rich region located at positions -41/-36 (GGGGCGGAA) that shared perfect homology to the consensus Sp-binding sequence (5'[G/T]GGGCGG [G/A] [G/A]-3') [8] (Figure 1). We also mapped a half-CRE-binding site (5'-CGTAA-3') immediately downstream (-23/-27) from the AP-1 element. Previous investigations from our laboratory have documented that estrogendependent stimulation of BRCA1 transcription required the assembly of an ER- $\alpha / p 300$ complex at the AP-1 site [33]. Because Sp-binding factors and CRE-binding factors have been shown to cooperate with $\mathrm{AP}-1$ in regulation of transcription, we examined the role played by these sites in controlling basal and estrogen-induced BRCA1 promoter activity. Breast cancer MCF-7 cells were transiently transfected with either a reporter-luciferase construct containing 1.7 kilobase of the wild-type BRCA1 promoter (pGL3BRCA1, -1552 to +140 
Figure 1

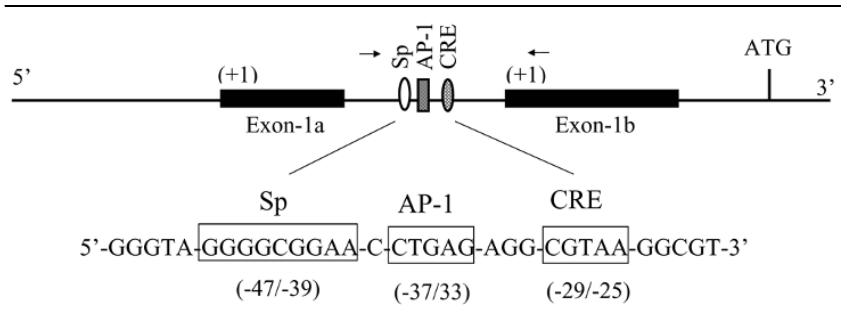

pGL3BRCA-1

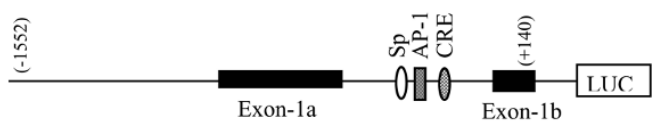

Regulatory sites of the human $B R C A 1$ promoter. A specificity protein (Sp) consensus site located upstream of an activator protein (AP)-1 element in the intervening sequence between exon-1a and exon-1b contributes to basal and estrogen-regulated transcription. A cAMP response element (CRE) site located downstream of the AP-1 contributes to basal regulation. Numbers in parenthesis for the Sp, AP-1, and CRE elements are base pairs from the initiation of transcription $(+1)$ on exon-1b. Nucleotides in boxes represent the sequence and spatial arrangement of the Sp, AP-1, and CRE domains. Arrows flanking the Sp/AP-1/CRE sites indicate the position of oligonucleotides used for real-time amplification after chromatin immunoprecipitation assays. The specific sequences of oligonucleotides are reported in the Materials and methods section. pGL3BRCA-1 represents a 1.692 kilobase BRCA1 promoter-luciferase (LUC) construct used in transfection studies. This promoter fragment extends from +140 base pairs to -1.552 kilobases from the +1 of exon- $1 \mathrm{~b}$. Details of the recruitment of a p300/ ER- $\alpha /$ SRC-1 complex to the AP-1 site are described elsewhere [33].

from +1 on exon-1b) or the pGL3BRCA1 construct carrying mutated Sp (pSpmut, 5'-GGctaaGAA-3') or CRE (pCREmut, 5'-CtgcA-3').
In MCF-7 cells transfected with pGL3-BRCA1, the treatment with estrogen induced (about 2.3-fold) BRCA1 promoter activity (Figure 2a) as compared with the control vehicle (DMEM), thus confirming the results of previous findings from our laboratory [34]. However, basal reporter activity was reduced by about $80 \%$ in MCF- 7 cells transfected with pSpmut. We observed a small increase $(1.2$-fold; $P<0.10)$ in $B R C A 1$ promoter activity upon treatment with estrogen, but this stimulation was considerably lower than that observed in cells transfected with the wild-type pGL3 BRCA1 promoter construct. Conversely, the mutation of the half CRE-binding site reduced by about $35 \%$ basal BRCA 1 promoter activity but did not interfere with estrogen-dependent activation (about 2.0-fold) of BRCA1 transcription. These results clearly highlighted the differential role of the Sp site compared with the half-CRE in the estrogen-dependent regulation of BRCA1. The efficacy of the transfection conditions and estrogen treatment were confirmed by the activation of transcription from a positive control promoter-luciferase construct harboring three consensus EREs (p3XERE; Figure 2b).

Based on this information, we analyzed the cooperativity between the Sp site and ER- $\alpha$ in estrogen-activation of the BRCA1 promoter. For these experiments, we selected the Hela cell line because it does not express ER- $\alpha$ and in previous studies [34], we successfully used this system to examine estrogen-regulated BRCA1 expression. In preliminary experiments, we ascertained that the cotransfection of Hela cells with p3XERE plus an empty vector (pCR3.1) did not yield measurable luciferase activity (Figure 3a). Compared with control vehicle (DMEM), the treatment with estrogen stimulated luciferase reporter activity in Hela cells cotransfected with a vector containing the cDNA cassette for ER- $\alpha(p E R \alpha)$

\section{Figure 2}
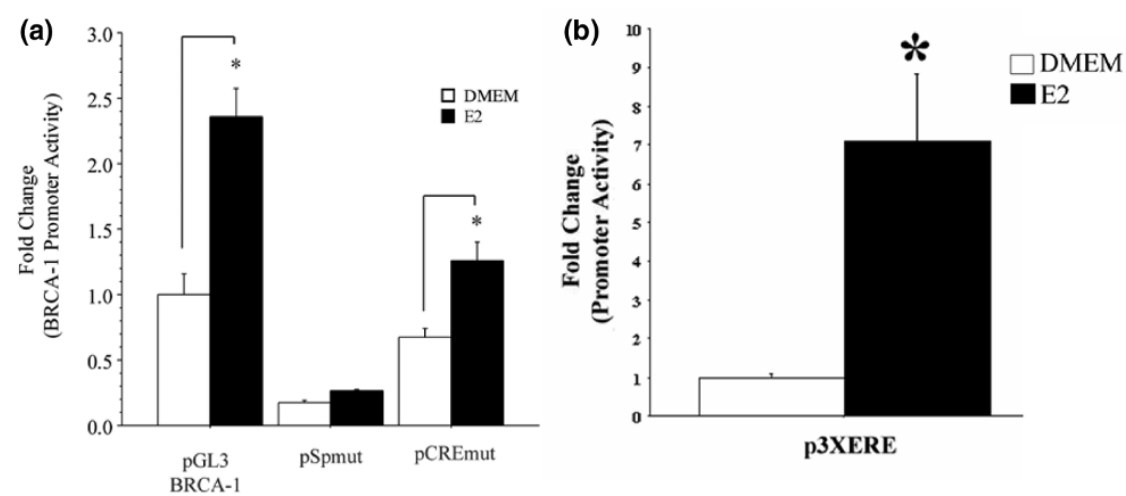

Sp site contributes to estrogen-dependent activation of the BRCA1 promoter in breast cancer MCF-7 cells. (a) Cells were precultured for 3 days in phenol red-free Dulbecco's modified Eagle's medium containing 5\% charcoal dextran-stripped fetal bovine serum (DMEM). Then, MCF-7 cells were transiently transfected with pGL3BRCA-1, pSpmut, or pCREmut. Cells were cultured in control vehicle (DMEM) or DMEM plus $10 \mathrm{nmol} / \mathrm{l} 17 \beta$-estradiol (E2) for 24 hours. (b) The treatment with E2 induced promoter activity from a positive control vector (p3xERE) transfected into MCF-7 cells. Bars represent mean luciferase units corrected for the internal control renilla \pm standard error from three independent experiments performed in quadruplicate. Asterisks indicate significant E2 activation $\left({ }^{\star} P<0.05\right)$ of promoter activity as compared with vehicle control $(D M E M)$ in cells transfected with pGL3BRCA-1, pSpmut, or pCREmut. 
plus p3XERE. These results confirmed the validity of the Hela cell line as a model system to study the regulation of BRCA1 by estrogen. In Hela cells transfected with pGL3BRCA1 plus the empty vector pCR3.1, the treatment with estrogen did not induce $B R C A 1$ promoter activity, although it was stimulated about 2.0-fold upon cotransfection with pER $\alpha$ (Figure 3b). In Hela cells cotransfected with the pSpmut construct alone, the basal promoter activity was reduced by $80 \%$, and estrogen activation was reduced in magnitude from 2.0-fold to 1.4-fold $(P<0.05)$. These results confirmed the important role of the crosstalk between the Sp site and ER- $\alpha$ in basal and estrogendependent regulation of BRCA1 transcription.

Next, we examined whether the Sp sequence influenced the recruitment of nuclear factors to the adjacent region of the $B R C A 1$ promoter harboring the AP-1 and CRE sites. BRCA1 oligonucleotides were coincubated with nuclear extracts obtained from MCF-7 cells cultured in control vehicle (DMEM) or in the presence of estrogen. The results of electromobility binding assays indicated that estrogen stimulated the binding of nuclear factors to the BRCA1 oligonucleotide harboring the AP-1 and CRE sites as evidenced by the increase in intensity of a DNA:protein complex (complex A; Figure 4a). However, we observed a striking increase in the intensity of this complex when nuclear extracts were coincubated with the BRCA1 oligonucleotide spanning the Sp-, AP-1, and CRE domains. Moreover, a second complex (complex B) of lower molecular weight was visualized by gel electrophoresis after incubation of nuclear extracts with the Sp/AP-1/CRE oligonucleotide. These results suggested that the complex $B$ probably comprised Sp proteins.

The role played by the Sp, AP-1, and CRE sites in the recruitment of nuclear factors was further investigated in competition studies with oligonucleotides harboring mutations for each site (Figure 4b). Compared with the wild-type labeled BRCA1 oligonucleotide, the incubation of nuclear extracts with cold wild-type Sp/AP-1/CRE oligonucleotide reduced the binding by nuclear factors (Figure 4c; lane 3). Conversely, the coincubation with cold oligonucleotides harboring mutated Sp, AP-1, or CRE differentially restored binding as compared to the wildtype labeled control (lanes 4 to 10). The most significant recovery of binding was observed with cold oligonucleotides mutated for Sp1 (lane 4), AP-1 (lane 5), and CREB (lanes 9 and 10). These cumulative data confirmed that the Sp, AP-1, and CRE sites played an important role in the formation of transcription complexes at this segment of the BRCA1 promoter.

To test whether the GC-box was a binding site for Sp factors and the ER- $\alpha$, we performed DNA-protein pull-down assays. Nuclear extracts harvested from control (DMEM) and estrogen-treated MCF-7 cells were incubated with BRCA1 oligonucleotides comprising the GC-box. Western blots revealed the constitutive occupancy of Sp-1, Sp3, and ER- $\alpha$ in control vehicle (DMEM) cells (Figure $5 \mathrm{a}$ ). For the $\mathrm{Sp} 3$ protein, we visualized two doublets of approximately 60 and $100 \mathrm{kDa}$, which represent the two short and two long forms from different translational initiation [38]. A similar pattern for Sp3 has been reported following Western blotting of cellular extracts obtained from MCF-7 cells $[39,40]$. The treatment with estrogen increased the binding levels of Sp1 and ER- $\alpha$ protein, but not the association of Sp3. The incubation of nuclear extracts with BRCA1 oligonucleotides mutated for the Sp-binding site (Spmut) reduced significantly the binding by $\mathrm{Sp} 1$ and Sp3, but minimally reduced the association of ER- $\alpha$. These results suggested that $\mathrm{Sp}$ binding was not required for the recruitment of the ER- $\alpha$ to the adjacent AP-1 site.

Figure 3
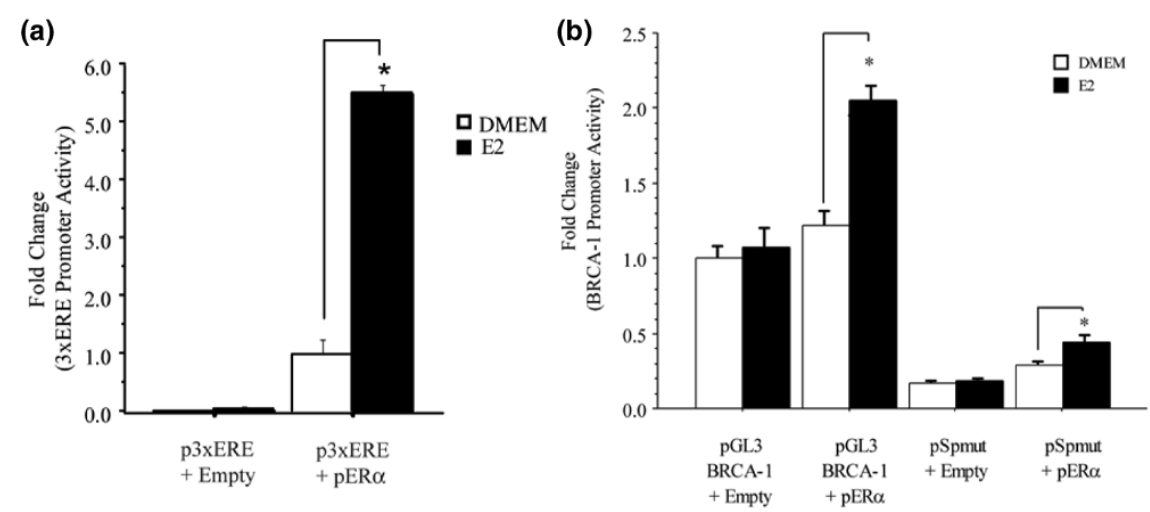

Sp site contributes to E2-dependent activation of the BRCA1 promoter in Hela cells expressing exogenous ER- $\alpha$. (a) Hela cells were cotransfected with pGL3BRCA-1 or pSpmut containing a mutated specificity protein (Sp) site plus the empty vector pCR3.1 or pER $\alpha$. Transfected cells were cultured in Dulbecco's modified Eagle's medium containing $5 \%$ charcoal dextran-stripped fetal bovine serum plus vehicle (DMEM) or DMEM plus 10 $\mathrm{nmol} / \mathrm{l} 17 \beta$-estradiol (E2) for 24 hours. (b) Hela cells were cotransfected with an empty plasmid (PCR3.1) plus p3xERE or p3XERE plus a vector containing the cDNA for the estrogen receptor $(E R)-\alpha(p E R \alpha)$. Bars represent mean luciferase units corrected for the internal control renilla \pm standard error from four independent experiments performed in quadruplicate. Asterisks indicate significant $\mathrm{E} 2$ activation $\left({ }^{*} P<0.05\right)$ of $B R C A 1$ promoter activity as compared with vehicle control (DMEM). 


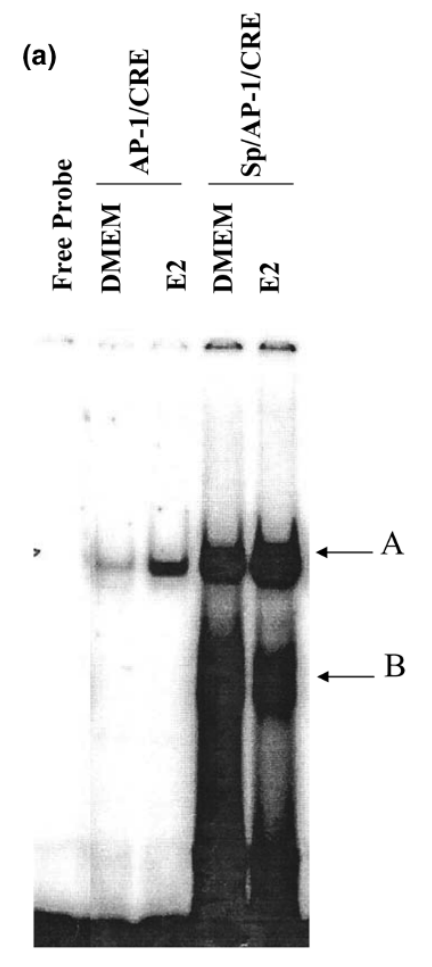

(c)

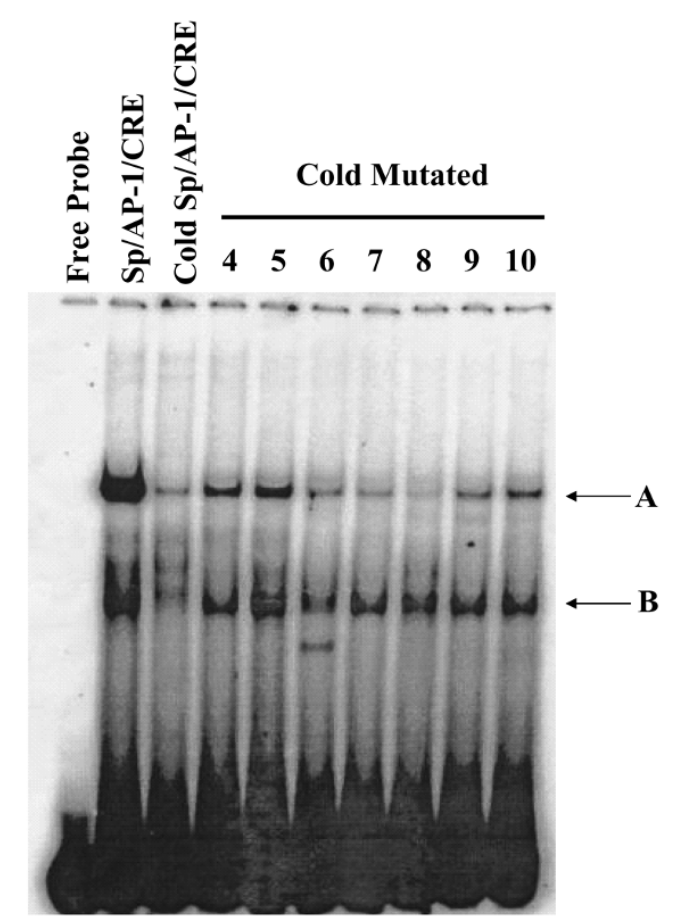

(b)

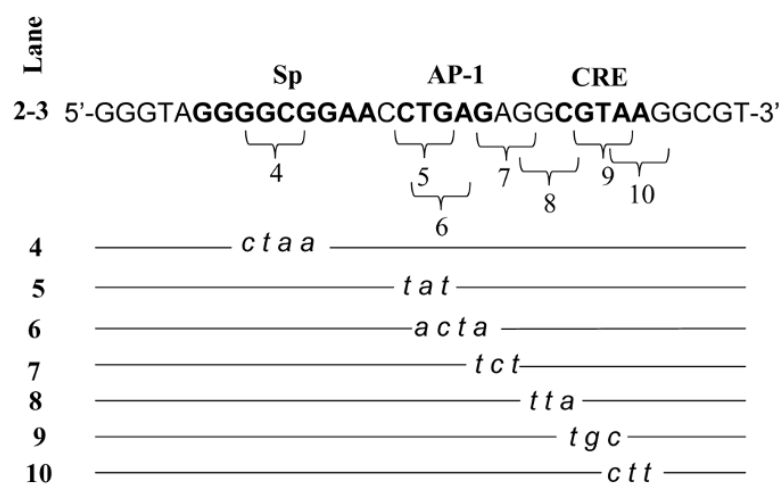

Sp site enhances the binding of nuclear proteins to the BRCA1 promoter. (a) Nuclear extracts normalized to $5 \mu \mathrm{g}$ of nuclear protein obtained from MCF-7 cells were incubated with ${ }^{32}$ P-labeled BRCA1 promoter oligonucleotides containing the activator protein (AP)-1/cAMP response element (CRE) or the Sp plus AP-1/CRE sites. MCF-7 cells were cultured for 3 hour in control vehicle (DMEM) or DMEM plus $10 \mathrm{nmol} / \mathrm{l} 17 \beta$-estradiol (E2). FP, free probe (lane 1). (b, c) In competition studies, nuclear extracts from MCF-7 cells cultured in the presence of E2 were incubated with ${ }^{32 P-}$ labeled BRCA1 oligonucleotides containing the wild-type specificity protein (Sp)/AP-1/CRE sequences in the absence (lane 2) or presence (100fold) of competing cold wild-type Sp/AP-1/CRE oligonucleotide (lane 3), or 100-fold of competing cold oligonucleotides mutated for Sp (lane 4), AP-1 (lanes 5 and 6), intervening sequences between AP-1 and CRE (lanes 7 and 8), and CRE (lanes 9 and 10). The arrows indicate the DNA:protein complexes visualized by electromobility binding assay.

Interactions of CREB proteins with the BRCA1 promoter region containing the Sp/AP-1/CRE sequences were investigated by gel electromobility shift assay. Nuclear proteins were incubated with the Sp1/CRE or Sp/Ap1/CRE oligonucleotides and, upon gel separation, we observed (Figure $5 \mathrm{~b}$ ) a pattern of retarded bands ( $A$ and $B$ ) that was similar to that depicted in Figure 4a. Upon coincubation with an antibody for
CREB, we visualized a slower migrating band suggesting this protein was associated with the Sp/CRE or Sp/AP-1/CRE oligonucleotide. These findings suggested that the $\mathrm{Sp}$ site or estrogen treatment did not influence the constitutive recruitment of CREB to the AP-1/CRE region. 

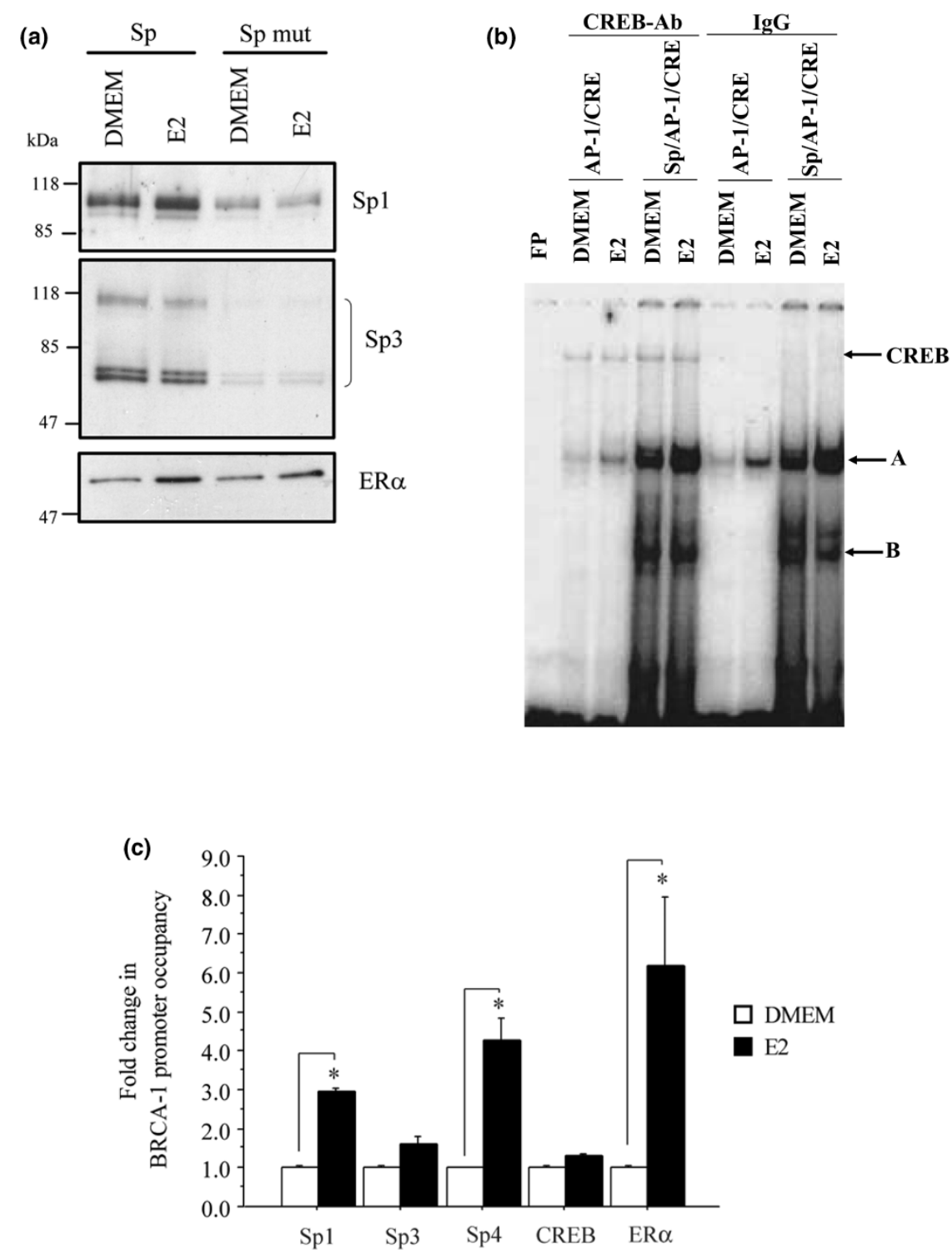

Estrogen stimulates the recruitment of Sp to a GC-rich region in the BRCA1 promoter. (a) Nuclear protein extracts $(200 \mu \mathrm{g})$ were obtained from MCF-7 cells cultured for 1.5 hours in Dulbecco's modified Eagle's medium containing $5 \%$ charcoal dextran-stripped fetal bovine serum containing vehicle (DMEM) or DMEM plus $10 \mathrm{nmol} / / 17 \beta$-estradiol (E2). DNA:protein complexes were generated by coincubation of nuclear extracts with the wild-type specificity protein (Sp)1/activator protein (AP)-1 oligonucleotide or the same oligonucleotide harboring a mutated Sp (Spmut) and separated by SDS-PAGE. The recruitment of Sp1, Sp3, or estrogen receptor (ER)- $\alpha$ was visualized by Western blotting with specific antibodies. Panels are representative of two independent experiments. Numbers represent molecular weight markers $(\mathrm{kDa})$. (b) Nuclear extracts normalized to $5 \mu \mathrm{g}$ of nuclear protein obtained from MCF-7 cells were incubated with ${ }^{32} \mathrm{P}$-labeled BRCA1 promoter oligonucleotides containing the AP-1/CRE or the Sp plus AP-1/CRE sites. MCF-7 cells were cultured for 3 hours in control vehicle (DMEM) or DMEM plus 10 nmol/l E2. FP, free probe (lane 1). Arrows indicate DNA:protein complexes (A and B) visualized by electromobility binding assay, and cAMP response element (CRE)-binding protein(CREB)/ DNA complexes supershifted with the CREB antibody. IgG, preimmune immunoglobulin control. (c) Basal and E2-induced recruitment of Sp1, Sp3, $\mathrm{Sp} 4$, and ER- $\alpha$ to the BRCA1 region containing the Sp, AP-1, and CRE sites was examined by chromatin immunoprecipitation assay followed by real-time PCR, as described in Materials and methods. The columns represent mean amplification products corrected for input \pm standard error (bars) and normalized to vehicle control (DMEM). Asterisks indicate statistically significant $(P<0.05)$ induction of factor recruitment by E2 as compared with vehicle control DMEM.

Further confirmation of the interactions of $\mathrm{Sp} 1, \mathrm{Sp} 3, \mathrm{Sp} 4$, ER$\alpha$, and CREB with the BRCA1 promoter was obtained by ChIP assay followed by real-time PCR analysis. The treatment with estrogen increased the occupancy of Sp1, Sp4, and ER- $\alpha$. The association of Sp3 and CREB was slightly increased by estrogen but not to a significant degree as compared with control DMEM (Figure 5c). These in vivo results complemented those obtained with DNA-pull down and electro- 
Figure 6

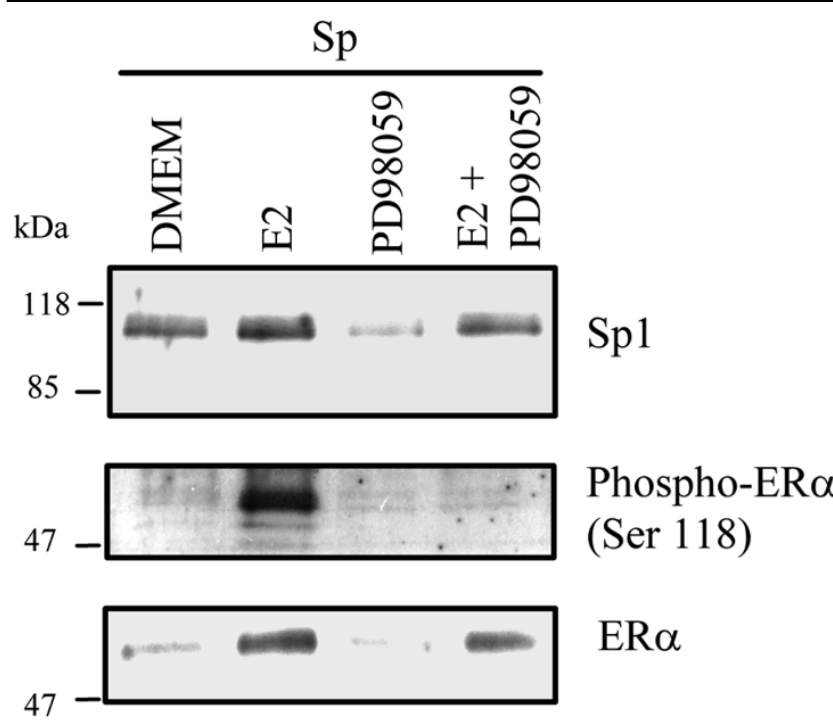

The MEK-1 inhibitor reduces the E2-induced binding of Sp1 and ER- $\alpha$ to the BRCA1 promoter. Nuclear protein extracts $(200 \mu \mathrm{g})$ were obtained from MCF-7 cells cultured for 1.5 hours in Dulbecco's modified Eagle's medium containing 5\% charcoal dextran-stripped fetal bovine serum containing vehicle (DMEM), or DMEM plus $10 \mathrm{nmol} / \mathrm{l}$ $17 \beta$-estradiol (E2), the MEK-1 (mitogen-activated protein kinase kinase-1) inhibitor PD98059, or E2 plus PD98059. DNA:protein complexes were generated by coincubation of nuclear extracts with the wild-type specificity protein (Sp) 1 /activator protein (AP)-1 oligonucleotide and separated by SDS-PAGE. The recruitment of Sp1, total ER$\alpha$, or phosphorylated ER- $\alpha$ was visualized by Western blotting with specific antibodies. Panels are representative of two independent experiments. Numbers indicate molecular weight markers $(\mathrm{kDa})$.

phoretic mobility shift assay experiments and corroborated the role of Sp1, Sp4, and ER- $\alpha$, and of CREB, respectively, in estrogen-regulated and basal BRCA1 transcription.

Exposure to estrogen has been shown to activate signaling cascades, including the MAPK kinase (MEK)/MAPK phosphorylation pathway that leads to phosphorylation of ER- $\alpha$ and related coactivators [41]. Therefore, we examined whether the MEK/MAPK pathway modulated the binding of Sp1 and ER- $\alpha$ to the BRCA1 promoter regions comprising the Sp and AP-1 sequences. Compared with MCF-7 cells cultured in control vehicle (DMEM), the treatment with estrogen stimulated the binding of Sp1 and ER- $\alpha$. This effect was counteracted by the cotreatment with estrogen plus the MEK1 kinase inhibitor PD98059 (Figure 6). The constitutive binding of Sp1 and ER$\alpha$ to the BRCA1 oligonucleotide was not influenced by the treatment with the PD98059 compound alone. As a positive control, we measured the association of phosphorylated ER- $\alpha$, whose binding to the BRCA1 oligonucleotide was reduced upon cotreatment with estrogen plus PD98059. These data suggested that the recruitment of Sp1 and ER- $\alpha$ to the region of the BRCA1 promoter comprising respectively the $\mathrm{Sp}$ and AP-1 binding elements was mediated through MEK-dependent activation of phosphorylation.

\section{Discussion}

Understanding how estrogen regulates BRCA1 expression may contribute to clarifying the role played BRCA1 under normal physiological conditions and pathophysiological perturbations that predispose to breast cancer. Previous studies from our laboratory documented that estrogen stimulated BRCA1 expression by inducing the recruitment of an ER- $\alpha / p 300 \mathrm{com}$ plex at an AP-1 site located close to the transcription start site of exon-1b [33]. The objective of this study was to define the role of $\mathrm{Sp}$ and $\mathrm{CRE}$ binding sites flanking the AP-1 element. The Sp-binding domain (5'-GGGGCGGAA-3') shared homology with the Sp1 consensus sequence (5'-[G/T]GGGCGG $\left.[G / A][G / A]-3^{\prime}\right)$. We found that mutation of the Sp site reduced basal and estrogen-dependent BRCA1 promoter activity in MCF-7 cells expressing endogenous ER- $\alpha$. In transfected Hela cells expressing exogenous ER- $\alpha$, the mutation of the $\mathrm{Sp}$ site led to a striking reduction in basal activity and reduced the estrogen activation. Cumulative data from DNA:protein binding and ChIP assays with BRCA1 oligonucleotides spanning the GC-rich and AP-1 domain suggested that estrogen stimulated the recruitment of Sp-1, Sp4, and ER$\alpha$ but did not affect the constitutive occupancy by Sp-3. Therefore, the estrogen-dependent activation of the proximal $B R C A 1$ promoter requires the intact Sp-binding site and appears to be mediated by increased occupancy of Sp1/Sp4 and ER- $\alpha$ as compared with Sp3.

Previous studies have documented that Sp3 can both enhance and inhibit transactivation. For example, the estrogendependent regulation of the VEGFR2 (vascular endothelial growth factor receptor 2) promoter was primarily regulated by ER- $\alpha / S p 3$ and ER- $\alpha / S p 4$, but not Sp1 [42]. Conversely, recent findings by the same group [43] documented a role for Sp1, Sp3, and Sp4 in regulating the VEGFR1 gene. Similarly, other reports corroborated a role for ER- $\alpha / S p 1$ in estrogendependent activation of the interleukin-1 $\alpha$ [44], insulin-like growth factor-1 [45], and cdc25A [46] gene promoter. Our results indicate that Sp1 and Sp4 play a specific role in estrogen-dependent regulation of BRCA1 transcription. Interestingly, mutation of the Sp site did not prevent the recruitment of the ER- $\alpha$ to BRCA1 oligonucleotides harboring the AP-1 domain, but it reduced transcriptional activity. These findings suggest that the estrogen-induced association of Sp1 and $\mathrm{Sp} 4$ at the GC-box may be functionally required for estrogenmediated transactivation of the BRCA1 promoter. The observation that the Sp-binding site enhanced the recruitment of nuclear proteins to BRCA1 oligonucleotides comprising the AP-1 and CRE elements suggests the Sp site may increase the stability of the ER- $\alpha / p 300$ complex recruited at the adjacent AP-1 site. In electromobility shift assays, we observed constitutive recruitment of CREB to a half-CRE (5'-CGTAA-3') located just downstream from the AP-1 motif. However, the constitutive occupancy by CREB was not altered by the estrogen treatment or the presence of the upstream Sp-binding site. 


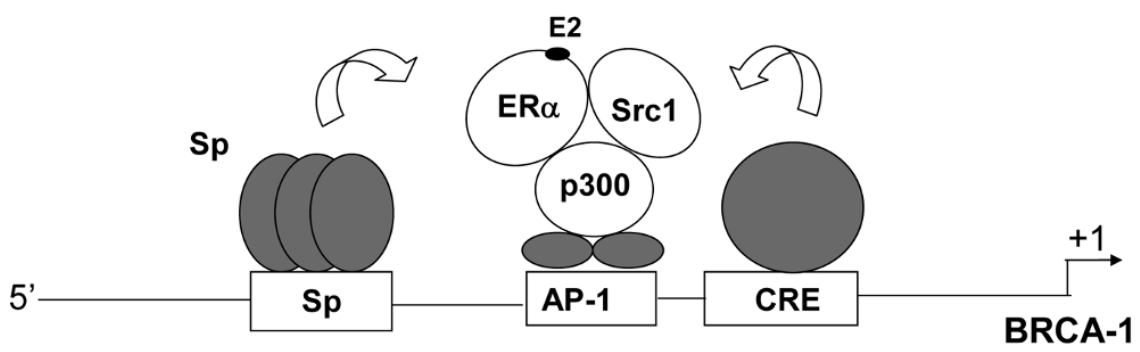

Proposed model for regulation of BRCA1 promoter activity. Results suggest that specificity proteins (Sps) contribute to basal and estrogen (E2)dependent transactivation of the BRCA1 promoter. The Sp-binding region is a target site for multiple Sps, including Sp1, Sp3, and Sp4. However, our data document increased recruitment of Sp1 and Sp4, but not Sp3, in response to E2. We also document the constitutive presence of cAMP response element (CRE)-binding protein (CREB) at the CRE site. Arrows indicate potential interactions of Sp and CREB factors with a p300/estrogen receptor (ER)- $\alpha$ transcription complex bound to an activator protein (AP)-1 (Jun/Fos) domain [33] harbored in the BRCA1 promoter. The TRANSFAC/Match transcription factor database [56] was used to map the Sp, AP-1, and CRE sites.

One possible model that integrates the current results is depicted in Figure 7. Activation of BRCA1 transcription by estrogen may be dependent on the increased interaction between Sp1, Sp4, and ER- $\alpha$ bound respectively to the Sp and $A P-1$ elements located in the proximal region of the $B R C A 1$ promoter. The occupancy by CREB at the half-CRE may serve as an ancillary factor that regulates constitutive activity. Interactions between ER- $\alpha, \mathrm{Sp} 1$, and Sp4 may trigger the subsequent recruitment of cofactors such as p300, which can function as a histone acetyltransferase [4], thus leading to estrogen-dependent activation of the BRCA1 gene. This model finds support in the experimental evidence that mutation of the $\mathrm{Sp}$ or AP-1 [33] element prevented the estrogendependent activation of the BRCA1 promoter.

The role of Sp proteins has been proposed to be promoter selective and dependent on the presence of flanking sequences [42]. The Sp1 factor has been demonstrated to interact physically with the ER- $\alpha$ and ER- $\beta$ [7], but only the ER$\alpha / \mathrm{Sp} 1$ interaction led to transcriptional activation of estrogenresponsive genes, such as $H s p 27$ [6], cad [47], and cathep$\sin -D$ [48]. Conversely, ER- $\alpha / \mathrm{Sp} 1$ interactions played a minimal role in activation of VEGFR2, and ER- $\alpha / \mathrm{Sp} 3$ and ER- $\alpha /$ Sp4 were critical factors [42]. In this study, we found that the treatment with estrogen increased the binding of the BRCA1 promoter by $\mathrm{Sp} 1$ and Sp4, but it did not alter the levels of bound Sp3. Similar to other genes involved in the oncogenic process $[49,50]$, the relative occupancy ratio between Sp proteins may play a critical role in transcriptional regulation of the BRCA1 gene. For example, elevated Sp3 levels have been shown to promote pancreatic and breast cancer cell growth by inhibiting transcription of growth inhibitory genes including thosse encoding the cyclin-dependent kinase inhibitor p27 and the type II receptor for transforming growth factor- $\beta$ $[8,51]$. Moreover, pancreatic cells transfected with small inhibitory RNA targeting Sp3 exhibited increased cell cycle distribution in $G_{0} / G_{1}$ and decreased distribution in $S$ phase [8]. Conversely, Sp1 sites were required for activation of the p21 promoter [52]. Based on the observation that Sp1-mediated transcriptional activation can be repressed by Sp3 [53], a shift in the Sp1/Sp4 versus Sp3 binding ratio may alter normal estrogen regulation of the BRCA1 gene. The model depicted in Figure 7 of cooperative interactions between DNA-bound Sp proteins and ER- $\alpha$ recruited at an AP- 1 site is similar to that reported for the E2F1 promoter, whose transactivation by the ER- $\alpha$ was found to be independent of direct interactions with promoter elements and was regulated by a multiprotein complex comprising ER/Sp1/NF-Y [54].

Regulation of $B R C A 1$ by estrogen may require activation of the MAPK cascade. Estrogen binds to the ER- $\alpha$ and stimulates MAPK-mediated phosphorylation of MEK1/2, which in turn phosphorylates ERK1/2 (extracellular signal regulated kinase1/2) [55]. The activated ERK1/2 may add phosphate groups to ER- $\alpha$ and $\mathrm{Sp}$ and modulate protein-DNA or protein-protein interactions of these factors at target promoters. Our results document that activation of the MEK pathway plays a key role in the estrogen-regulated recruitment of Sp1 and phosphorylated ER- $\alpha$ to the BRCA1 promoter.

\section{Conclusion}

We report that estrogen-dependent activation of BRCA1 transcription is mediated by a GC-rich region, which is a binding target for Sp proteins. The Sp-binding motif is located in close proximity to an AP-1 site, which is a target for an ER- $\alpha / p 300$ complex. Estrogen stimulates the recruitment of Sp1 and Sp4, but not Sp3, to the GC-rich region. We also provide evidence that the AP-1 site is flanked by a CRE-binding domain that regulates constitutive expression. The close proximity of the Sp, AP-1, and CRE-binding sites may be important for the recruitment of transcriptional complexes and regulation of basal and estrogen-dependent BRCA1 transcription. Finally, we provide evidence that the MAPK pathway regulates the association of Sp1 and ER- $\alpha$ with the BRCA1 promoter. Overall, these results offer new evidence for both genomic and nongenomic regulation of BRCA1 transcription activity by estrogen. 


\section{Competing interests}

The authors declare that they have no competing interests.

\section{Authors' contributions}

$\mathrm{JKH}, \mathrm{SCD}$, and DFR conceived the study. JKH conducted the mutational studies. SCD conducted the DNA-pull down assays, Western blot analyses, and ChIP studies. SSM conducted the binding and electromobility shift assays. MOK and JKH performed transfection studies. JKH, SCD, SSM, and DFR contributed to the drafting of the manuscript, which was approved by all authors.

\section{Acknowledgements}

This work was supported by NIH Grant ES009966 (DFR), fellowships from Graduate Training Program T32 ES-07091-24 (JKH and SCD), and a grant (100116) from the Arizona Biomedical Research Commission (DFR) and the Susan G. Komen Breast Cancer Foundation (DFR) (BCTR0707643).

\section{References}

1. Levin $E$ : Integration of the extranuclear and nuclear actions of estrogen. Mol Endocrinol 2005, 19:1951-1959.

2. Kushner P, Agard D, Feng W, Lopez G, Schiau A, Uht R, Webb P, Greene G: Oestrogen receptor function at a classical and alternative response elements. Novartis Found Symp 2000, 230:20-26.

3. McKenna N, O'Malley B: Combinatorial control of gene expression by nuclear receptors and coregulators. Cell 2002, 108:465-474.

4. Ogryzko V, Schiltz R, Russanova V, Howard B, Nakatani Y: The transcription coactivators $\mathrm{p} 300$ and CBP are histone acetyltransferases. Cell 1996, 87:953-959.

5. Paech K, Webb P, Kuiper G, Nilsson S, Gustafsson J, Kushner P, Scanlan T: Differential ligand activation of estrogen receptors ERalpha and ERbeta at AP1 sites. Science 1997, 277:1508-1510.

6. Porter W, Saville B, Hoivik D, Safe S: Functional synergy between the transcription factor $\mathrm{Sp} 1$ and the estrogen receptor. Mol Endocrinol 1997, 11:1569-1580.

7. Saville B, Wormke M, Wang F, Nguyen T, Enmark E, Kuiper G, Gustafsson J, Safe S: Ligand-, cell-, and estrogen receptor subtype (alpha/beta)-dependent activation at GC-rich (Sp1) promoter elements. J Biol Chem 2000, 275:5379-5387.

8. Safe S, Abdelrahim M: Sp transcription factor family and its role in cancer. Eur J Cancer 2005, 41:2438-2448.

9. Yu B, Datta P, Bagchi S: Stability of the Sp3-DNA complex is promoter-specific: Sp3 efficiently competes with $\mathrm{Sp} 1$ for binding to promoters containing multiple Sp-sites. Nucleic Acids Res 2003, 31:5368-5376.

10. Sapetschnig A, Koch F, Rischitor G, Mennenga T, Suske G: Complexity of translationally controlled transcription factor Sp3 isoform expression. J Biol Chem 2004, 279:42095-42105.

11. Bouwman P, Philipsen S: Regulation of the activity of Sp1related transcription factors. Mol Cell Endocrinol 2002, 195:27-38.

12. Marin M, Karis A, Visser $P$, Grosveld F, Philipsen S: Transcription factor Sp1 is essential for early embryonic development but dispensable for cell growth and differentiation. Cell 1997, 89:619-628.

13. Bouwman $P$, Gollner H, Elsasser H, Eckhoff G, Karis A, Grosveld F, Philipsen S, Suske G: Transcription factor Sp3 is essential for post-natal survival and late tooth development. EMBO J 2000, 19:655-661.

14. Gollner H, Dani C, Phillips B, Philipsen S, Suske G: Impaired ossification in mice lacking the transcription factor Sp3. Mech Dev 2001, 106:77-83.

15. Supp D, Witte D, Branford W, Smith E, Potter S: Sp4, a member of the Sp1-family of zinc finger transcription factors, is required for normal murine growth, viability, and male fertility. Dev Biol 1996, 176:284-299.
16. Wang L, Wei D, Huang S, Peng Z, Le X, Wu TT, Yao J, Ajani J, Xie $\mathrm{K}$ : Transcription factor Sp1 expression is a significant predictor of survival in human gastric cancer. Clin Cancer Res 2003, 9:6371-6380.

17. Yao J, Wang L, Wei $D$, Gong $W$, Hassan $M$, Wu T, Mansfield $P$, Ajani J, Xie K: Association between expression of transcription factor Sp1 and increased vascular endothelial growth factor expression, advanced stage, and poor survival in patients with resected gastric cancer. Clin Cancer Res 2004, 10:4109-4117.

18. Shi Q, Le X, Abbruzzese J, Peng Z, Qian C, Tang H, Xiong Q, Wang B, Li X, Xie K: Constitutive Sp1 activity is essential for differential constitutive expression of vascular endothelial growth factor in human pancreatic adenocarcinoma. Cancer Res 2001, 61:4143-4154.

19. Zannetti A, Del Vecchio S, Carriero M, Fonti R, Franco P, Botti G D'Aiuto G, Stoppelli M, Salvatore M: Coordinate up-regulation of Sp1 DNA-binding activity and urokinase receptor expression in breast carcinoma. Cancer Res 2000, 60:1546-1551.

20. Bilsland A, Stevenson K, Atkinson S, Kolch W, Keith W: Transcriptional repression of telomerase RNA gene expression by c-Jun-NH2-kinase and Sp1/Sp3. Cancer Res 2006, 66:1363-1370.

21. Merchant J, Du M, Todisco A: Sp1 phosphprylation by Erk 2 stimulates DNA binding. Biochem Biophys Res Commun 1999, 254:454-461.

22. Wang Y, Cortez D, Yazdi P, Neff N, Elledge S, Qin J: BASC, a super complex of BRCA-1 associated proteins involved in the recognition and repair of aberrant DNA structures. Genes Dev 2000, 14:927-939.

23. Moynahan M, Chiu J, Koller B, Jasin M: Brca1 controls homologydirected DNA repair. Mol Cell 1999, 4:511-518.

24. Tibbetts $R$, Cortez $D$, Brumbaugh $K$ : Functional interactions between BRCA1 and the checkpoint kinase ATR during genotoxic stress. Genes Dev 2000, 14:2989-3002.

25. Taylor J, Lymboura M, Pace P, A'hern R, Desai A, Shousha S, Coombes R, Ali S: An important role for BRCA1 in breast cancer progression is indicated by its loss in a large proportion of non-familial breast cancers. Int J Cancer 1998, 79:334-342.

26. Wilson CA, Ramos L, Villaseñor MR, Anders KH, Press MF, Clarke K, Karlan B, Chen JJ, Scully R, Livingston D, Zuch RH, Kanter MH, Cohen S, Calzone FJ, Slamon DJ: Localization of human BRCA1 and its loss in high grade, non-inherited breast carcinomas. Nat Genet 1999, 21:236-240.

27. Gudas J, Nguyen H, Li T, Cowan K: Hormone-dependent regulation of BRCA1 in human breast cancer cells. Cancer Res 1995, 55:4561-4565.

28. Gudas J, Li T, Nguyen H, Jensen D, Rauscher F, Cowan K: Cell cycle regulation of BRCA1 messenger RNA in human breast epithelial cells. Cell Growth Differ 1996, 7:717-723.

29. Romagnolo D, Annab L, Thompson T, Risinger J, Terry L, Barrett J, Afshari C: Estrogen upregulation of BRCA1 expression with no effect on localization. Mol Carcinog 1998, 22:102-109.

30. Xu C, Brown M, Chambers J, Griffiths B, Nicolai H, Solomon E: Distinct transcription start sites generate two forms of BRCA1 mRNA. Hum Mol Genet 1995, 4:2259-2264.

31. Fan S, Wang JA, Yuan RQ, Ma YX, Meng Q, Erdos MR, Brody LC, Goldberg ID, Rosen EM: BRCA1 inhibition of estrogen receptor signaling in transfected cells. Science 1999, 284:1354-1386.

32. Welcsh PL, Lee MK, Gonzalez-Hernandez RM, Black DJ, Mahadevappa M, Swisher EM, Warrington JA, King MC: BRCA1 transcriptionally regulates genes involved in breast tumorigenesis. Proc Natl Acad Sci USA 2002, 99:7560-7565.

33. Jeffy B, Hockings J, Kemp M, Morgan S, Hager J, Beliakoff J, Whitesell L, Bowden T, Romagnolo D: An estrogen receptoralpha/p300 complex activates the BRCA-1 promoter at an AP1 site that binds Jun/Fos transcription factors: repressive effects of p53 on BRCA-1 transcription. Neoplasia 2005, 7:873-882.

34. Hockings J, Thorne P, Kemp M, Morgan S, Selmin O, Romagnolo $D$ : The ligand status of the aromatic hydrocarbon receptor modulates transcriptional activation of the BRCA-1 promoter by estrogen. Cancer Res 2006, 66:2224-2232.

35. Jeffy B, Chirnomas R, Chen E, Gudas J, Romagnolo D: Activation of the aromatic hydrocarbon receptor pathway is not sufficient for transcriptional repression of BRCA-1: requirements for metabolism of benzo[a]pyrene to BPDE. Cancer Res 2002, 62:113-121. 
36. Degner S, Kemp M, Bowden T, Romagnolo D: Conjugated linoleic acid attenuates cyclooxygenase-2 transcriptional activity via an anti-AP-1 mechanism in MCF-7 breast cancer cells. J Nutr 2006, 136:421-427.

37. Selmin O, Thorne PA, Blachere FM, Johnson PD, Romagnolo DF: Transcriptional activation of the membrane-bound progesterone receptor (mPR) by dioxin, in endocrine-responsive tissues. Mol Reprod Dev 2005, 70:166-174.

38. Sapetschnig A, Koch F, Rischitor G, Mennenga T, Suske G: Complexity of translationally controlled transcription factor Sp3 isoform expression. J Biol Chem 2004, 279:42095-42105

39. He S, Sun J-M, Li L, Davie JR: Differential intranuclear organization of transcription factors Sp1 and Sp3. Mol Biol Cell 2005 , 16:4073-4083.

40. Khan S, Wu F, Liu S, Wu Q, Safe S: Role of specificity protein transcription factors in estrogen-induced gene expression in MCF-7 breast cancer cells. J Mol Endocrinol 2007, 39:289-304.

41. Collins P, Webb C: Estrogen hits the surface. Nat Med 1999, 5:1130-1131.

42. Higgins KJ, Liu S, Abdelrahim M, Yoon K, Vanderlaag K, Porter W, Metz RP, Safe S: Vascular endothelial growth factor receptor-2 expression is induced by $17 \beta$-estradiol in ZR-75 breast cancer cells by estrogen receptor $\alpha /$ Sp proteins. Endocrinology 2006, 147:3285-3295.

43. Abdelrahim M, Baker $\mathrm{CH}$, Abbruzzese JL, Sheikh-Hamad D, Liu S, Cho SD, Yoon K, Safe S: Regulation of vascular endolethial growth factor receptor-1 expression by specific proteins 1, 3, and 4 in pancreatic cancer cells. Cancer Res 67:3286-3294.

44. Itoh Y, Hayashi H, Miyazawa K, Kojima S, Akahoshi T, Onozaki K: 17beta-estradiol induces II1 $\alpha$ gene expression in rheumatoid fibroblast-like synovial cells through estrogen receptor $\alpha$ $(E R \alpha)$ and augmentation of transcriptional activity of Sp1 by dissociating histone acetylase 2 from ER . J Immunol 2007, 178:3059-3066.

45. Maor S, Mayer D, Yarden RI, Lee AV, Sarfstein R, Werner H, Papa $M Z$ : Estrogen receptor regulates insulin-like growth factor-1 receptor gene expression in breast tumor cells: involvement of transcription factor Sp1. J Endocrinol 2006, 191:605-612.

46. Ru LW, Chen CC, Safe S: 17beta estradiol (E2) induces cdc25A gene expression in breast cancer cells by genomic and non-genomic pathways. J Cell Biochem 2006, 99:209-220.

47. Khan S, Abdelrahim M, Samudio I, Safe S: Estrogen receptor/ Sp1 complexes are required for induction of cad gene expression by 17 beta-estradiol in breast cancer cells. Endocrinology 2003, 144:2325-2335.

48. Duan R, Porter W, Safe S: Estrogen-induced c-fos protooncogene expression in MCF-7 human breast cancer cells: role of estrogen receptor Sp1 complex formation. Endocrinology 1998, 139:1981-1990.

49. Tang S, Bhatia B, Zhou J, Maldonado C, Chandra D, Kim E, Fischer S, Butler A, Friedman S, Tang D: Evidence that Sp1 positively and Sp3 negatively regulate and androgen does not directly regulate functional tumor suppressor 15-lipoxygenase 2 (15LOX2) gene expression in normal human prostate epithelial cells. Oncogene 2004, 23:6942-6953.

50. Hasleton $M$, lbbitt $J$, Hurst $\mathrm{H}$ : Characterization of the human activator protein-2gamma (AP-2gamma) gene: control of expression by $\mathrm{Sp} 1 / \mathrm{Sp} 3$ in breast tumour cells. Biochem $J$ 2003, 373:925-932.

51. Ammanamanchi $\mathrm{S}$, Brattain $\mathrm{M}: \mathrm{Sp} 3$ is a transcriptional repressor of transforming growth factor-beta receptors. I Biol Chem 2001, 276:3348-3352.

52. De Siervi A, Marinissen M, Diggs J, Wang X, Pages G, Senderowicz A: Transcriptional activation of p21(waf1/cip1) by alkylphospholipids: role of the mitogen-activated protein kinase pathway in the transactivation of the human p21(waf1/cip1) promoter by Sp1. Cancer Res 2004, 64:743-750.

53. Hagen G, Muller S, Beato M, Suske G: Sp1-mediated transcriptional activation is repressed by Sp3. EMBO J 1994, 13:3843-3851.

54. Wang W, Dong L, Saville B, Safe S: Transcriptional activation of E2F1 gene expression by $17 \mathrm{~b}$-estradiol in MCF-7 cells is regulated by NF-Y-Sp1/estrogen receptor interactions. Mol Endocrinol 1999, 13:1373-1387.

55. Harrington W, Kim S, Funk C, Madak-Erdogan Z, Schiff R, Katzenellenbogen J, Katzenellenbogen B: Estrogen dendrimer conjugates that preferentially activate extranuclear, nong- enomic versus genomic pathways of estrogen action. Mol Endocrinol 2006, 20:491-502.

56. TRANSFAC/Match transcription factor database [http:// www.gene-regulation.com] 